\title{
Sensitivity of a Single Coil Electromagnetic Sensor for Non-contact Monitoring of Breathing
}

\author{
Rosalyn Seeton and Andy Adler
}

\begin{abstract}
We design and analyse a flexible non-contact electromagnetic sensor for monitoring breathing. This paper presents the sensor design and the theoretical model for the expected sensitivity from which we optimize design components. The primary application of this device is in non-invasive sleep monitoring and it has the potential to be useful for detection of both adult and infant apnea. The conductivity changes of the lungs during breathing are monitored by inducing eddy currents within them. The sensor consists of a small coil that acts as both a transmitter and a receiver. The theoretical sensitivity of the sensor to breathing changes is calculated and corresponds within the assumptions of the model to the measured results.
\end{abstract}

\section{INTRODUCTION}

There is significant interest in monitoring breathing during sleep to detect and measure sleep apnea. Adult sleep apnea is a widespread condition that decreases sleep quality and it is being linked with systemic hypertension, cardiovascular disease, stroke, and abnormal glucose metabolism [1]. Apnea in infants is associated with Sudden Infant Death Syndrome [2]. Sleep monitoring and apnea sensing require a method of supervising a patient without interfering with their natural state. In other words, an ideal sensor for these applications would be non-invasive, minimally restrictive, robust enough to compensate for patient movement, and would function without relying on patient cooperation. Electromagnetic sensors can provide unobtrusive monitoring of lung activity and are therefore good potential candidates for applications such these.

Eddy current sensors have an inductive coil that carries a time varying current, which produces a changing magnetic field. Conductive materials subjected to this dynamic magnetic field experience eddy currents to counteract the changes in flux. This effect can be detected in a receiving coil and the resulting measurement is a function of the conductivity of the material.

The more well-known uses of electromagnetic sensors that rely on eddy currents are for positioning or proximity measurement, and for non-destructive testing of conductive materials. However, the activities of the heart and lungs also cause conductivity changes within the body that can be detected by an electromagnetic sensor. Sensors have been designed for this purpose with multiple coils, which are either perpendicular to each other [3] or coaxial [4][5]. Alternatively, one coil can be used as both the transmitter and receiver [6][7][8]. Different coil sensors have been designed to calculate volumes [9] (such as heart ejection volume [10])

The authors are with the Department of Systems and Computer Engineering, Carleton University, Ottawa, Canada, (seeton, adler)@sce.carleton.ca or image the distribution of conductivity [11]. Difficulties arise with these applications from the fact that the eddy current signals are so small compared to the driving signals.

This paper continues our previous investigation [8] in which we seek to design a sensor system that is flexible enough to be placed in a mattress. Such a system will experience deformations, and must not depend on a constant geometry between drive and receive coils. In order to accomplish this, we do not attempt to calculate an image of the conductivity distribution, but rather a single parameter which correlates to breathing and heart activity. We use a flexible coil which acts as both transmitter and receiver. The advantage of the single flexible coil measurement system is that an array of sensors, each with a single sensor, can be embedded into a mattress cover, which can be placed between the subject and the mattress for sleep studies. The flexible coils are able to tolerate bending due to the subject weight and movement, and provide a continuous monitoring of the subject's breathing pattern.

The goal of this paper is twofold: to develop a theoretical model of our sensor's sensitivity in order to evaluate the feasibility for lung or heart monitoring, and to develop an approach to optimizing the circuit design for maximal sensitivity by selecting components based on our theoretical model.

\section{METHODS}

\section{A. Experimental Investigation}

We conducted a simple experiment with a subject lying on a mattress in which a single sensor was positioned under the thorax such that the coil was flat with the axis pointing to the lung. The subject was asked to perform breathing manoeuvres such as tidal breathing, forced expiration, forced inspiration, and breath holding. Flow measurements were taken concurrently using a pneumotachograph connected to a pressure transducer in order to track the breathing patterns. Experimental details are given in [8].

The block diagram of our system is shown in Fig. 1. As the subject's heart beats and lungs inflate and deflate the conductivity in the thorax varies. To simplify our analysis we take the body to be a constant arbitrary shape, which we can therefore ignore, and we model breathing as a change in conductivity over a spherical volume $V$ representing the lung. The physiological effects are seen by the sensor as a change in equivalent impedance of our coil and this alters the oscillation frequency. The frequency of the oscillator from a subject performing breathing manoeuvres is shown in Fig. 4. 


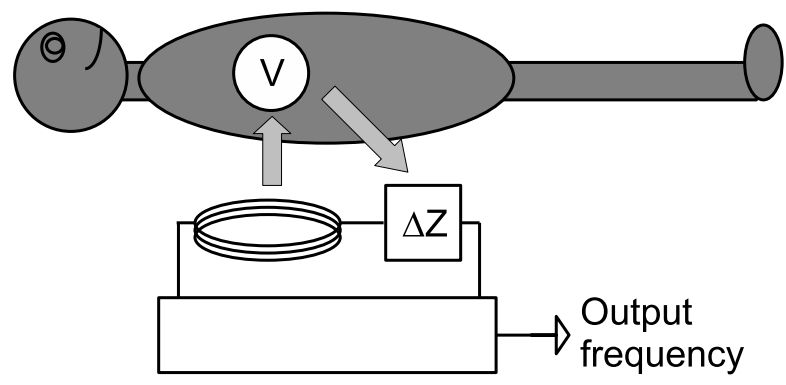

Fig. 1. Experimental setup

\section{B. Electronic design of the sensor}

Our sensor is constructed using a single coil within a circuit which oscillates due to an odd number of inverters (CMOS 74HC04) as shown in Fig. 2. Oscillations in the coil create eddy currents in conductive tissue which are seen by the circuit as a change in coil impedance from $j \omega L$ to $j \omega L+\Delta Z$ as represented by an additional dashed resistor. Oscillations also cause displacement currents in tissue with non-zero permittivity, which are seen by the circuit as a change in coil inductance. However, since the lung permittivity is low at the frequency of interest, we do not consider this effect.

The frequency of oscillations of this circuit with the added impedance is approximated as follows:

$$
f \approx \frac{1}{2 \ln 3} \frac{R_{1}+\Delta Z}{L},
$$

where we have assumed that $R_{2}$ is large and does not draw much current. We want to maximize our ratio of change in frequency to base frequency,

$$
\frac{\Delta f}{f}=\frac{\Delta Z}{R_{1}},
$$

so we conclude that we must minimize $R_{1}$ in the circuit. However, there is a tradeoff as minimizing $R_{1}$ also decreases the operating frequency and $\Delta Z$ is proportional to the square of the frequency. To keep the frequency constant and decrease $R_{1}$, the inductance $L$ would need to decrease. In a given sensor there are practical limits to the size of $L$.

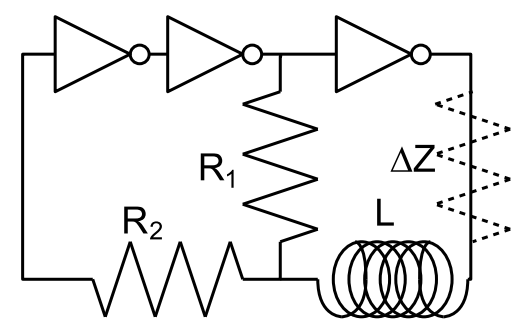

Fig. 2. Electronic circuit for single coil bistable LR oscillator

\section{Theoretical model of a current carrying coil}

In this section, we derive the theoretical model for a single circular coil with a sphere of conductive material, which represents the lung, centered on the axis of the coil.

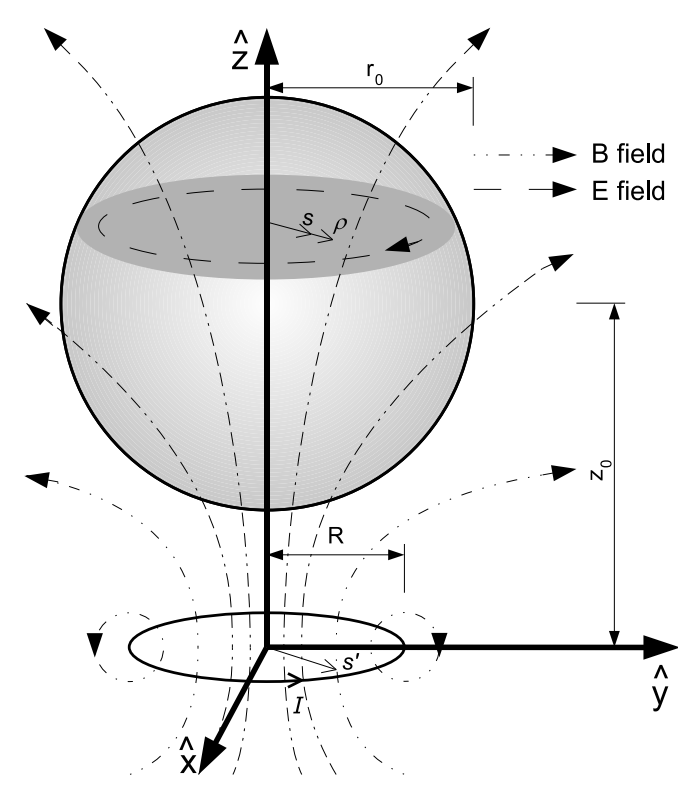

Fig. 3. Coil carrying current I centered on $x-y$ axis with a conductive sphere above it

Our analysis follows the same approach as [6] but for the geometry of a sphere. The sensitivity of the coil is then calculated in terms of the change in frequency caused by the conductive material. We assume that the permeability and the permittivity remain constant at their free space values so that the only change is the conductivity of our material.

The theoretical model is derived from quasistatic electromagnetic theory. The magnetic field from a coil of $N$ turns, carrying a current $I$ centered on the x-y axis as seen in Fig. 3 can be described by the Biot-Savart law:

$$
\mathbf{B}=\frac{\mu_{0} N I}{4 \pi} \int \frac{d \mathbf{l} \times \hat{r}}{r^{2}},
$$

where $\mu_{0}=4 \pi \times 10^{-7}\left(N / A^{2}\right)$ is the permeability of free space. If the coil has radius $R$ and we choose a point located at a height $z$ above the coil, at a radius $s$ from the $\mathrm{z}$ axis, the magnetic field is given in cylindrical coordinates by:

$$
\mathbf{B}(s, z, t)=\frac{\mu_{0} N I R}{4 \pi} \int_{0}^{2 \pi} \frac{z d \phi \hat{\mathbf{s}}+(R-s \cos \phi) d \phi \hat{\mathbf{z}}}{\left(s^{2}+R^{2}+z^{2}-2 s R \cos \phi\right)^{\frac{3}{2}}} .
$$

Since the radial component of the magnetic field will not contribute to any change in flux in the coil, we are only concerned with the $\hat{\mathbf{z}}$ component which we call $\mathbf{B}_{\mathbf{z}}(s, z, t)=$ $I \mathbf{B}_{\mathbf{z}}(s, z)$ and we define:

$$
\mathbf{B}_{\mathbf{z}}(s, z)=\frac{\mu_{0} N R}{4 \pi} \int_{0}^{2 \pi} \frac{(R-s \cos \phi) d \phi \hat{\mathbf{z}}}{\left(s^{2}+R^{2}+z^{2}-2 s R \cos \phi\right)^{\frac{3}{2}}} .
$$

The solution to $\mathbf{B}_{\mathbf{z}}(s, z)$ takes the form of complete elliptic integrals of the first and second kind. The electric field at our chosen point can then be calculated from the flux $\Phi$ using the integral form of Faraday's Law $\oint \mathbf{E} \cdot d \mathbf{l}=-\frac{d \Phi}{d t}=-\int \frac{d \mathbf{B}}{d t} \cdot d \mathbf{a}$ 
as follows:

$$
\begin{aligned}
\mathbf{E}(\rho, z, t) & =\frac{-1}{2 \pi \rho} \frac{d I}{d t} \int_{0}^{\rho} \int_{0}^{2 \pi} s \mathbf{B}_{\mathbf{z}}(s, z) d \phi d s \hat{\phi} \\
& =\frac{-1}{\rho} \frac{d I}{d t} \int_{0}^{\rho} s \mathbf{B}_{\mathbf{z}}(s, z) d s \hat{\phi} .
\end{aligned}
$$

We apply Ohm's Law to find the current density at point $(s, z)$. We assume that the material has isotropic and homogeneous conductivity and permittivity so that $\mathbf{E} \| \mathbf{J}$. Then the current density is:

$$
\begin{aligned}
\mathbf{J}(\rho, z, t) & =\left(\sigma+j \omega \epsilon_{0}\right) \mathbf{E}(\rho, z, t) \\
& =-\frac{\left(\sigma+j \omega \epsilon_{0}\right)}{\rho} \frac{d I}{d t} \int_{0}^{\rho} s \mathbf{B}_{\mathbf{z}}(s, z) d s \hat{\phi},
\end{aligned}
$$

where $\sigma$ is the conductivity and $\epsilon_{0}=8.85 \times 10^{-12} \frac{C^{2}}{N m^{2}}$ is the permittivity of free space. The induced eddy current through a surface is then given by integrating the current density over the area of interest $\mathbf{I}=\int \mathbf{J} \cdot d \mathbf{a}$. We want to model our conductive material as a sphere and do this by breaking the sphere into very thin disks, which are parallel to the coil, and summing the contributions from each of the disks. Each of those conductive disks, in turn, can be modeled as concentric eddy current carrying loops which we sum over the radius of the disk. So an element of current can be described in differential form:

$$
d \mathbf{I}(\rho, z, t)=-\left(\sigma+j \omega \epsilon_{0}\right) \frac{d I}{d t} d z \frac{d \rho}{\rho} \int_{0}^{\rho} s \mathbf{B}_{\mathbf{z}}(s, z) d s \hat{\phi} .
$$

We now project backwards and calculate the magnetic field induced at the plane of the coil by each element of eddy current. Once again, we only care about the component in the axial direction. The induced magnetic field in the $x-y$ plane at a radius $s^{\prime}$ is then:

$$
\mathbf{B}_{\mathbf{z}}\left(s^{\prime}, z, t\right)=\int d \mathbf{I}(\rho, z, t) \mathbf{B}_{\mathbf{z}}\left(s^{\prime}, z\right) \hat{\mathbf{z}},
$$

where our original $\mathbf{B}_{\mathbf{z}}(s, z)$ expression is used with the following substitutions: $R=\rho, s=s^{\prime}$ and $N=1$. We define the radial limit of each disk as $r_{z}=\sqrt{r_{0}^{2}-\left(z_{0}-z\right)^{2}}$ and we sum the contributions from each disk by integrating from $z_{1}=z_{0}-r_{0}$ to $z_{2}=z_{0}+r_{0}$, where $z_{0}$ is the height of the centre of the sphere above the coil and $r_{0}$ is the radius of the sphere. If we were to look at the less geometrically complex case of a cylinder, the radial limit would simply be the radius of the cylinder and the axial limits would be assigned the height of the base and top of the cylinder. Returning to the spherical source, our magnetic field induced at the coil from all disks is then:

$\mathbf{B}_{\mathbf{z}}\left(s^{\prime}, z, t\right)=\int_{z_{1}}^{z_{2}} \int_{0}^{r_{z}} \int_{0}^{\rho} \frac{s C}{\rho} \mathbf{B}_{\mathbf{z}}(s, z) \mathbf{B}_{\mathbf{z}}\left(s^{\prime}, z\right) d s d \rho d z \hat{\mathbf{z}}$, where constant $C=-\left(\sigma+j \omega \epsilon_{0}\right) \frac{d I}{d t}$.

The induced magnetic field causes a change in the magnetic flux inside a loop of the coil given by:

$$
\begin{aligned}
\Phi & =\int \mathbf{B}_{\mathbf{z}}\left(s^{\prime}, z, t\right) \cdot d \mathbf{a} \\
& =\int_{0}^{R} \int_{0}^{2 \pi} s^{\prime} \mathbf{B}_{\mathbf{z}}\left(s^{\prime}, z, t\right) d \phi d s^{\prime} .
\end{aligned}
$$

This manifests as an electromotive force proportional to the number of loops in the coil:

$$
\begin{aligned}
\varepsilon & =-N \frac{d \Phi}{d t} \\
& =C \int_{0}^{R} \int_{z_{1}}^{z_{2}} \int_{0}^{r_{z}} \int_{0}^{\rho} F d s d \rho d z d s^{\prime} \hat{\phi}, \\
\text { where } C & =2 \pi N\left(\sigma+j \omega \epsilon_{0}\right) \frac{d^{2} I}{d t^{2}} \\
\text { and } F & =\frac{s s^{\prime}}{\rho} \mathbf{B}_{\mathbf{z}}(s, z) \mathbf{B}_{\mathbf{z}}\left(s^{\prime}, z\right) .
\end{aligned}
$$

We assume that the current is sinusoidal and given by $I=A e^{j \omega t}$. As such, the second derivative is $-\omega^{2} I$ so our constant in the above expression simplifies to $C=$ $-2 \pi \omega^{2} N I\left(\sigma+j \omega \epsilon_{0}\right)$. The change in inductance of the coil is $L=\frac{N \Phi}{I}=\frac{-\varepsilon}{j \omega I}$. Alternatively, the EMF can be seen by the coil as an effective series impedance $Z=\frac{-\varepsilon}{I}$.

In order to solve Eqn. (2) it is necessary to integrate numerically. This was solved using Matlab by integrating Eqn. (1) for $\mathbf{B}_{\mathbf{z}}(s, z)$ symbolically and then performing the rest of the integration numerically using adaptive Simpson quadrature by nesting functions as follows:

$$
\begin{aligned}
\text { Impedance } & =-\omega^{2} N \cdot \text { Flux } \\
\text { Flux } & =\int_{0}^{R} \int_{0}^{2 \pi} s^{\prime} \cdot \text { BField }\left(s^{\prime}\right) d \phi d s^{\prime} \\
\text { BField }\left(s^{\prime}\right) & =\int_{z_{1}}^{z_{2}} \int_{0}^{r_{z}} B\left(s^{\prime}, z, \rho, 1\right) \cdot J(\rho, z) d \rho d z \\
J(\rho, z) & =\left(\sigma+j \omega \epsilon_{0}\right) \cdot \text { EField }(\rho, z) \\
\text { EField }(\rho, z) & =-\frac{1}{2 \pi \rho} \int_{0}^{\rho} \int_{0}^{2 \pi} s \cdot B(s, z, R, N) d \phi d s \\
B(s, z, R, N) & =\mathbf{B}_{\mathbf{z}}(s, z) \text { in elliptic integral form }
\end{aligned}
$$

In the implementation of this algorithm, we divide the double integral of the flux into a nested function as well. We can model breathing by modeling the lung as a sphere, which expands on inspiration. The impedance change in our circuit from a subject performing a total lung capacity breathing manoeuvre is calculated by taking the volume of one lung to be 2.5 litres, which corresponds to a radius $r_{0}=0.0842 \mathrm{~m}$ for one lung, and the difference in conductivity to be $\sigma=$ $0.18 \mathrm{~S} / \mathrm{m}$. Our coil has radius $R=0.035 \mathrm{~m}$ and $N=10$ loops and the oscillator outputs at a frequency of $5.8 \mathrm{MHz}$. Using this expected change in impedance and calculations from our electronic circuit, we derive the expected change in frequency from conductive objects located on axis.

\section{RESULTS}

Fig. 4 shows the circuit output frequency for a subject during an episode of maximal lung manoeuvres (from [8]). Changes in sensor output correspond to inspiration and expiration manoeuvres as shown by the pneumotach output. There is a change in frequency $\Delta f \approx 0.002 \mathrm{MHz}$ from time $t \approx 18$ seconds to time $t \approx 23$ seconds in Fig. 4 which corresponds to the subject inspiring to total lung capacity after residual volume. Based on these measurements, and 

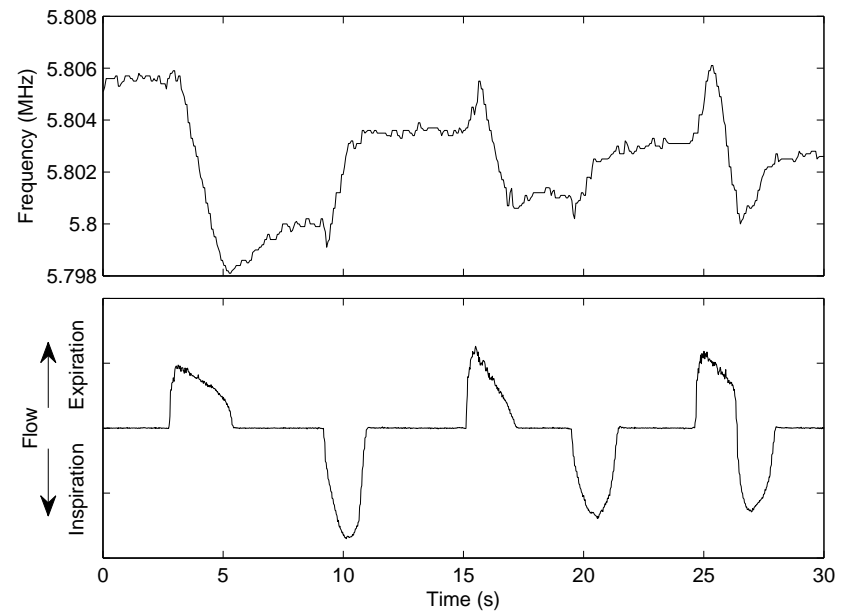

Fig. 4. Measured frequency output from subject performing breathing manoeuvres and corresponding flow output measured using a pneumotach

circuit values of $R_{1}=180 \Omega$ and $L=14 \mu H$, we make the following calculations:

$$
\Delta Z=\frac{\Delta f}{f} R \approx .062 \Omega
$$

We plot the theoretical $\Delta V$ as a function of distance $r_{0}$ in Fig. 5 and our calculated value of eddy current induced impedance change corresponds to a distance $z_{0} \approx 0.105 \mathrm{~m}$. Given an estimate $r_{0}$ of a sphere of inspired air for one lung, the edge closest to the sensor is at a distance of just over $2 \mathrm{~cm}$. The tissue thickness between skin and lung at this subjects back is approximately $5 \mathrm{~cm}$, so this value is in the right range, especially considering the number of assumptions in our theoretical model.

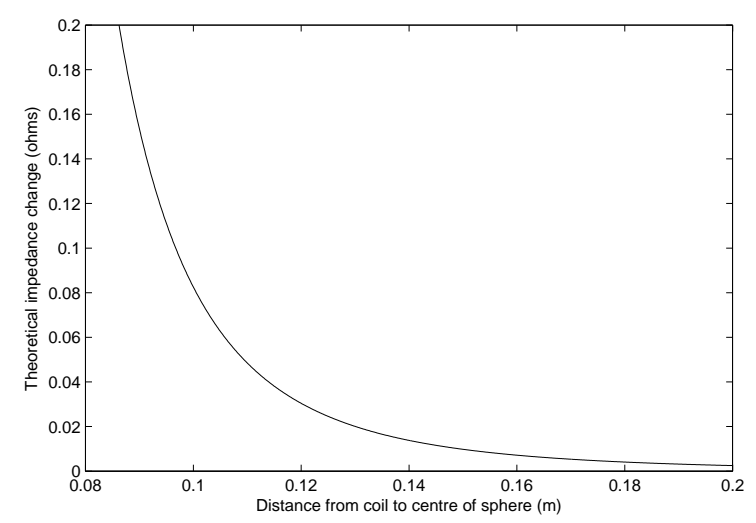

Fig. 5. Theoretical impedance $\Delta Z$ as a function of distance $z_{0}$ from the centre of a spherical object of radius $r_{0}=0.0842 \mathrm{~m}$

\section{Discussion}

This paper presents the design and electromagnetic analysis of a non-contact sensor for monitoring breathing. First, we present the sensor design and measurement configuration. Next, we have shown the theoretical model for a sphere of conductive material located on axis above a coil. Results compare measurements in healthy volunteers to the theoretical predictions and show plausible agreement. We note several assumptions and inaccuracies, mainly: 1) artifacts caused by patient movement are significant as electromagnetic measurements of this kind suffer from low frequency changes; 2) there is non-uniform conductivity distribution within the lung. Additionally, the positioning and orientation of the sensor relative to the body will affect the output since the signal has such a strong dependence on the distance between sensor and source. As we move our conductive object off axis the problem becomes much more complicated as we lose the cylindrical symmetry and the eddy currents will no longer be confined to traveling in concentric circles. Furthermore, the measured output that we analysed was from maximal breathing manoeuvres, whereas practical applications for sleep monitoring would consist of shallower, more continuous breaths. The signal strength could potentially be improved by a demodulation scheme and by reducing sources of noise.

Overall, this work makes the following contributions: 1) a model of sensitivity of single coil electromagnetic sensors, 2) an approach to optimize the circuit components to maximize sensitivity.

\section{ACKNOWLEDGMENTS}

This work was supported by a grant from NSERC Canada.

\section{REFERENCES}

[1] N.M. Punjabi, The epidemiology of adult obstructive sleep apnea, Proc. Am. Thorac. Soc., vol. 5, no. 2, 2008, pp 136-143.

[2] A. Steinschneider, Prolonged apnea and the sudden infant death syndrome: Clinical and laboratory observations, Pediatrics, vol. 50, no. 4, 1972, pp 646-654.

[3] C.H. Igney, S. Watson, R.J. Williams, H. Griffiths and O. Dössel, Design and performance of a planar-array MIT system with normal sensor alignment, Physiol. Meas., vol. 26, 2005, pp 263278.

[4] P.P. Tarjan and R. McFee, Electrodeless Measurement of the Effective Resistivity of the Human Torso and Head by Magnetic Induction, IEEE Transactions on Bio-medical Engineering, vol. BME-15, no. 4, 1968, pp 266-278.

[5] J. Rosell, R. Casañas and H. Scharfetter, Sensitivity maps and system requirements for magnetic induction tomography using a planar gradiometer, Physiol. Meas., vol. 22, 2001, pp 121-130.

[6] L. W. Hart, H. W. Ko, J. H. Meyer Jr., D. P. Vasholz and R. Joseph, A Noninvasive Electromagnetic Conductivity Sensor for Biomedical Applications, IEEE Transactions on Biomedical Engineering, vol. 35, no. 12, 1988, pp 1011-1022.

[7] R. Guardo, G. Charron, Y. Goussard and P. Savard, "Contactless Measurement of Thoracic Conductivity Changes by Magnetic Induction", in Proceedings - 19th International Conference - IEEE/EMBS, Chicago, IL, 1997, pp. 2450-2453.

[8] A. Richer and A. Adler, "Eddy Current Based Flexible Sensor for Contactless Measurement of Breathing", in Instrumentation and Measurement Technology Conference, Ottawa, Canada, 2005, pp.257-260.

[9] M. Steffe, A. Aleksandrowicz, and A. Leonhardt, Mobile Noncontact Monitoring of Heart and Lung Activity, IEEE Transactions on Biomedical Circuits and Systems, vol. 1, no. 4, 2007, pp 250-257.

[10] R. Guardo, S. Trudelle, A. Adler, C. Boulay, and P. Savard, "Contactless Recording of Cardiac Related Thoracic Conductivity Changes", in Proceedings - 17th International Conference - IEEE/EMBS, 1995, pp. 1581-1582.

[11] S. Watson, A. Morris, R. J. Williams, H. Griffiths and W, Gough, A primary field compensation scheme for planar array magnetic induction tomography, Physiol. Meas., vol. 25, 2004, pp 271-279. 